\title{
Results of frozen elephant trunk from the international E-vita Open registry
}

\author{
Konstantinos Tsagakis ${ }^{1}$, Davide Pacini ${ }^{2}$, Martin Grabenwöger ${ }^{3}$, Michael A. Borger ${ }^{4}$, Nora Goebel ${ }^{5}$, \\ Wolfgang Hemmer ${ }^{6}$, Alvaro Laranjeira Santos ${ }^{7}$, Thanos Sioris ${ }^{8}$, Kazimierz Widenka ${ }^{9}$, Petar Risteski ${ }^{10}$, \\ Jorge Mascaro $^{11}$, Igor Rudez ${ }^{12}$, Andreas Zierer ${ }^{13}$, Carlos A. Mestres ${ }^{14,15}$, Arjang Ruhparwar ${ }^{1}$, \\ Roberto Di Bartolomeo ${ }^{2}$, Heinz Jakob ${ }^{1}$
}

${ }^{1}$ Department of Thoracic and Cardiovascular Surgery, West German Heart and Vascular Center Essen, University Hospital of Essen, Essen, Germany; ${ }^{2}$ Department of Cardiac Surgery, S. Orsola Hospital, University of Bologna, Bologna, Italy; ${ }^{3}$ Department of Cardiovascular Surgery, Hospital Hietzing, Vienna, Austria; ${ }^{4}$ Department of Cardiac Surgery, Leipzig Heart Center, University of Leipzig, Leipzig, Germany; ${ }^{5}$ Department of Cardiac and Vascular Surgery, Robert Bosch Hospital, Stuttgart, Germany; ${ }^{6}$ Department of Cardiac Surgery, Sana Cardiac Surgery Stuttgart GmbH, Stuttgart, Germany; ${ }^{7}$ Department of Cardiothoracic Surgery, Hospital de Santa Marta, CHLC, Lisbon, Portugal; ${ }^{8}$ Tampere University Hospital Heart Center, Tampere, Finland; ${ }^{9}$ Szpital Wojewódzki N2, Oddział Kardiochirurgii, Rzeszów, Poland; ${ }^{10}$ Department of Thoracic and Cardiovascular Surgery, University Hospital Frankfurt am Main, Frankfurt am Main, Germany; ${ }^{11}$ Department of Cardiothoracic Surgery, University Hospital Birmingham NHS Foundation Trust, Birmingham, UK; ${ }^{12}$ Department of Cardiac and Transplant Surgery, University Hospital Dubrava, Zagreb, Croatia; ${ }^{13}$ Department of Thoracic and Cardiovascular Surgery, Faculty of Medicine, Kepler University Hospital Linz, Johannes Kepler University Linz, Linz, Austria; ${ }^{14}$ Department of Cardio Vascular Surgery, Hospital Clinico, University of Barcelona, Barcelona, Spain; ${ }^{15}$ Department of Cardiovascular Surgery, University Hospital Zürich, Zürich, Switzerland

Correspondence to: Konstantinos Tsagakis, MD, PhD. Department of Thoracic and Cardiovascular Surgery, West German Heart and Vascular Center Essen, University Hospital of Essen, Hufelandstr. 55, 45122 Essen, Germany. Email: konstantinos.tsagakis@uk-essen.de.

Background: Over the years, frozen elephant trunk (FET) has become the treatment of choice for multisegmental thoracic aortic disease. This multicenter study presents the evolution of FET results using the E-vita Open hybrid graft with respect to institutional experience and time.

Methods: The data of International E-vita Open registry were studied according to the institutional experience of the participating centers (high- versus low-volume centers) and according to the evolution of FET treatment during time ( $1^{\text {st }}$ period, 2005-2011 versus $2^{\text {nd }}$ period, 2012-2018). Overall, 1,165 patients were enrolled in the study with a wide variety of multisegmental thoracic aortic pathologies and aortic emergencies. Participating centers determined their own surgical protocol.

Results: The overall 30-day mortality was $12 \%$. Short- and long-term survival were higher in high- versus low-volume centers $\left(\mathrm{P}=0.048\right.$ and $\mathrm{P}=0.013$, respectively). In the $2^{\text {nd }}$ time period, cerebral complications were reduced significantly $(\mathrm{P}=0.015)$. Incidence of permanent spinal cord-related symptoms was reduced to $3 \%$ in the $2^{\text {nd }}$ time period, but did not reach statistical significance. Hypothermic circulatory arrest time $(\mathrm{P}<0.001)$ and incidence of postoperative temporary renal replacement therapy $(\mathrm{P}=0.008)$ were significantly reduced in the $2^{\text {nd }}$ time period. Ten-year survival and freedom from aortic-related death rates were $46.6 \%$ and $85.7 \%$, respectively, for the entire group. The freedom from distal aortic re-interventions for a new or progressive residual aortic disease was $76.0 \%$.

Conclusions: Evolution of FET arch repair techniques with the E-vita Open graft and increasing institutional experience were associated with improved results. Progression of residual aortic disease makes close follow-up with aortic imaging mandatory in such patients.

Keywords: Frozen elephant trunk (FET); aortic dissection; aortic aneurysm; aortic arch

Submitted Feb 19, 2020. Accepted for publication Mar 31, 2020.

doi: 10.21037/acs-2020-fet-25

View this article at: http://dx.doi.org/10.21037/acs-2020-fet-25 


\section{Introduction}

Aortic arch disease (i.e., aneurysm or dissection) is commonly combined with additional pathology of the descending aorta. In this setting, the frozen elephant trunk (FET) technique offers the option for a combined treatment of the aortic arch and the descending aorta in one step (1). It is based on the use of a hybrid graft, which enables the surgical replacement of the aortic arch and the endovascular exclusion of the descending aortic disease via a single median sternotomy. Although this surgical approach is considered a major surgery with substantial morbidity and mortality, FET currently represents the treatment of choice in many institutions. The hybrid graft can be applied in a wide variety of aortic arch pathologies with very few restrictions regarding aortic arch anatomy, including aortic emergencies (2-5).

In Europe, the first serial experience with FET technique was started in 2005 using the E-vita Open ${ }^{\mathrm{TM}}$ hybrid graft (Jotec/CryoLife, Kennesaw, GA, USA), the first commercially available FET prosthesis (6). Several lessons were learned during the early experience including patient selection, graft sizing and deployment, as well as improved organ protection techniques (7). The aim of this multicenter study was to evaluate the influence of increased institutional experience with FET treatment, as well as the effect of time, on postoperative results.

\section{Methods}

Data were recorded anonymously among participating centers in the database of the International E-vita Open registry and were evaluated for completion. Centers with incomplete documentation were excluded from the statistical analysis. Between 2005 and 2018, 19 European centers provided complete data on 1,165 patients, which form the basis of this study. The indications for surgery were acute or chronic type A, non-A-non-B, and type B aortic dissection, as well as degenerative aneurysmal disease of the distal arch or thoracoabdominal aorta. All patients underwent FET surgery with the E-vita Open ${ }^{\mathrm{TM}}$ and from 2009 onwards with the next generation of the graft, the E-vita Open Plus ${ }^{\mathrm{TM}}$.

In order to evaluate the hospital experience of FET technique, referring centers that performed more than fifty FET operations or more than ten patients per year, were considered high-volume centers. Six centers were classified as high-volume and thirteen as low-volume. In an attempt to differentiate and capture the learning experience, data were also divided into two key time intervals: period 1 from 2005-2011 and period 2 from 2012-2018.

Participating centers determined their own surgical protocol, including cannulation technique, temperature management, circulatory arrest technique, perfusion management, myocardial protection, graft sizing, and arch repair technique (e.g., en bloc versus separate branch reimplantation, debranching of the left subclavian, etc.). More detailed descriptions of these techniques can be found in previous publications (8-10). All patients in the current study underwent open arch repair via median sternotomy under hypothermic circulatory arrest and selective cerebral perfusion. Decision of graft sizing and planning of surgery for one- or two-staged procedures were performed by the referral centers.

\section{E-vita Open and E-vita Open Plus}

The E-vita Open and the E-vita Open Plus graft consists of two vascular prosthetic components as a continuous tube: a proximal surgical prosthesis for the arch and a distal self-expandable stent graft with Z-nitinol stents for the descending aorta. The graft is delivered by a flexible atraumatic introducer through the open aortic arch into the lumen of the descending aorta, and is deployed with the proximal arch graft crimped and invaginated within the stent graft lumen (6). This design enables the fixation of the graft in the aortic arch level by surgical suture, similar to the modified elephant trunk technique (11). After suture fixation, the incorporated arch prosthesis is released from the stent graft lumen into the arch position and is used for the replacement of the arch. In E-vita Open Plus, a collar has been added between the two components of the graft in order to facilitate suture fixation to the aortic wall. The tubular form of the prosthesis allows the implantation of the supra-aortic arteries en-bloc, as well as separately with or without interposition grafts. The E-vita Open stent graft diameters are available between 20 and $40 \mathrm{~mm}$, and the stent graft length is between 130 and $160 \mathrm{~mm}$.

\section{Definitions}

Over time, the technique of arch repair was changed in most participating centers, with debranching techniques for rerouting of the left subclavian artery in order to proximalize the stent graft fixation to zone 2 or even more proximally. In order to simplify the presentation of the arch 


\begin{tabular}{|c|c|c|c|c|c|c|c|}
\hline Patients & $\begin{array}{l}\text { Overall } \\
(\mathrm{N}=1,165)\end{array}$ & Low $(\mathrm{N}=334)$ & High $(\mathrm{N}=831)$ & $\mathrm{P}$ & First $(\mathrm{N}=385)$ & Second $(N=780)$ & $\mathrm{P}$ \\
\hline Age & $61.6 \pm 12$ & $63.1 \pm 12$ & $61.0 \pm 12$ & 0.007 & $61.4 \pm 12$ & $61.7 \pm 12$ & 0.664 \\
\hline Male & 786 [68] & $208[62]$ & $578[70]$ & 0.019 & $275[71]$ & $511[66]$ & 0.046 \\
\hline Acute dissection & $444[38]$ & 88 [26] & $356[43]$ & $<0.001$ & $134[35]$ & $310[40]$ & 0.109 \\
\hline Chronic dissection & 329 [28] & 98 [29] & $231[28]$ & 0.615 & $125[32]$ & 204 [26] & 0.027 \\
\hline Aneurysm & $392[34]$ & $148[44]$ & 244 [29] & $<0.001$ & $126[33]$ & 266 [34] & 0.693 \\
\hline Coronary artery disease & 209 [18] & 49 [15] & $160[19]$ & 0.076 & 64 [17] & $145[19]$ & 0.465 \\
\hline Previous endo- treatment & $68[6]$ & $18[5]$ & $50[6]$ & 0.783 & $24[6]$ & $44[6]$ & 0.691 \\
\hline Renal insufficiency & $91[8]$ & $14[4]$ & $77[9]$ & 0.003 & $37[10]$ & $54[7]$ & 0.131 \\
\hline COPD & $241[21]$ & $48[14]$ & 193 [23] & 0.001 & $62[16]$ & 179 [23] & 0.007 \\
\hline History of stroke & $76[7]$ & $24[7]$ & $52[6]$ & 0.600 & $22[6]$ & $54[7]$ & 0.528 \\
\hline Peripheral artery disease & $152[13]$ & 62 [19] & 90 [11] & 0.001 & 70 [18] & 82 [11] & $<0.001$ \\
\hline
\end{tabular}

Data are presented as $\mathrm{n}[\%]$. COPD, chronic obstructive pulmonary disease.

arteries revascularization technique, a common reinsertion of the brachiocephalic trunk and the left carotid artery was called "en-bloc" technique. "Separate reimplantation" technique represents only those patients with separate reinsertion of all three arch arteries, directly or by interposition grafts.

Mortality is presented as 30-day mortality or in-hospital mortality. Stroke is reported as a new permanent cerebral event, or a temporary event in case of neurological recovery. Spinal cord ischemic injury includes both paraplegia and paraparesis, and is presented as a permanent event in case of persisting neurological deficit. Spinal cord events with regressive neurological symptoms up to complete recovery were defined as temporary.

\section{Statistical analysis}

Data analysis was performed with the SPSS Package 26. Categorical and continuous variables are presented in percent and mean \pm standard deviation (SD), respectively. The two-sided Fischer exact test or the independent Student's $t$-test, if appropriate, were used for calculation of statistical significance $(\mathrm{P}<0.05)$ between groups.
Follow-up was provided by the referral centers for 989 patients at a mean \pm SD time of $3.2 \pm 2.8$ years postoperatively. Estimated calculations of survival and freedom from re-interventions in follow-up were performed by the Kaplan-Meier analysis. Cox regression analysis was used to evaluate the impact of centers and learning experience to survival and the freedom of aortic reinterventions. According to the number of patients available at follow-up, the statistical analysis for differences between groups was performed at 6 years. Aortic-related death within follow-up was defined as a death related to proximal or distal aortic disease, secondary aortic treatment, and unknown cause of death.

\section{Results}

\section{Patient characteristics}

The average age of patients was $61.6 \pm 12$ years and $67.5 \%$ were male (Table 1). In high-volume centers, more patients underwent surgery for acute aortic dissection $(\mathrm{P}<0.001)$ and they suffered from more comorbidities including aortic valve regurgitation $(\mathrm{P}=0.002)$, renal insufficiency $(\mathrm{P}=0.003)$ 


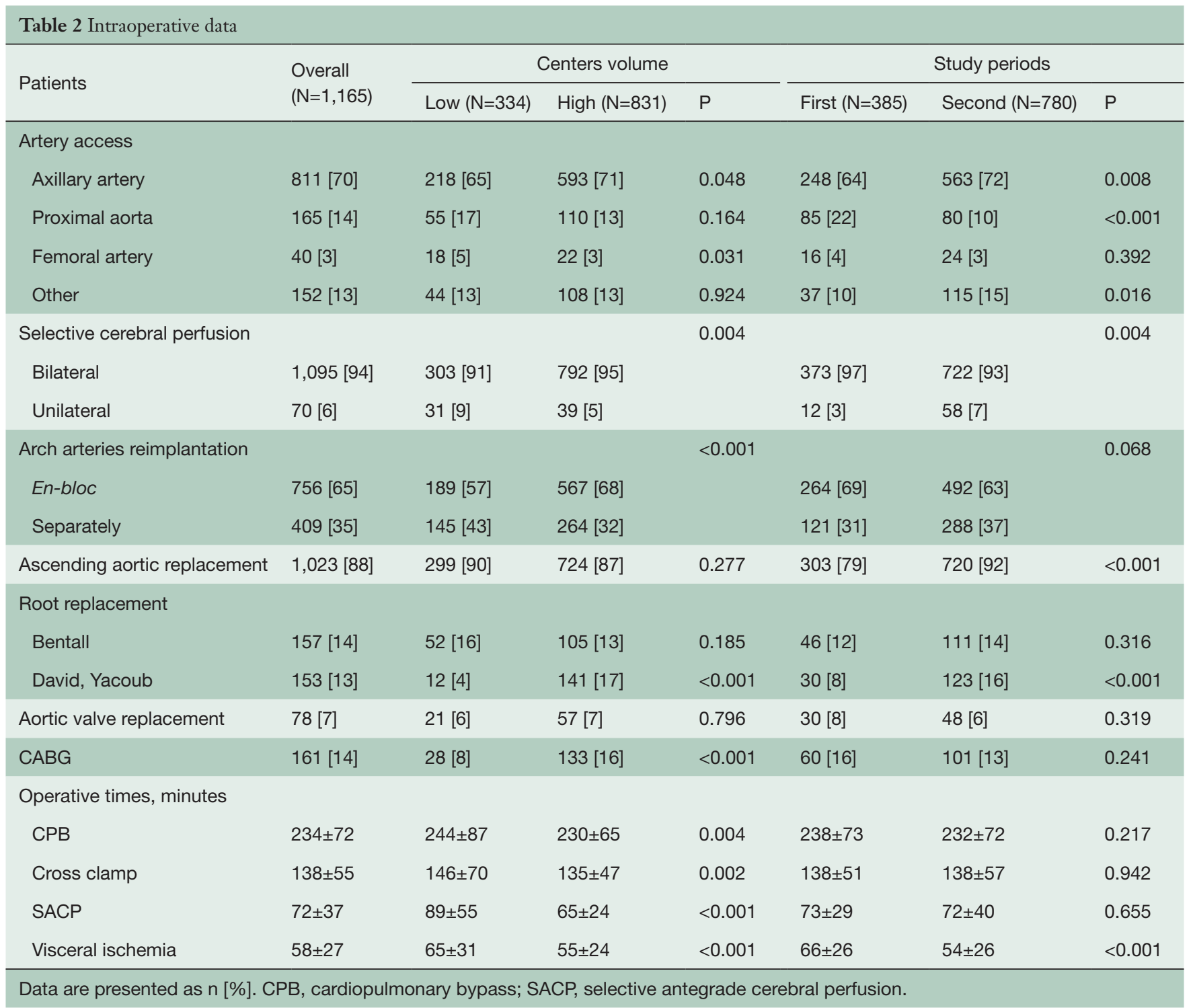

and pulmonary disease $(\mathrm{P}=0.001)$. By contrast, low-volume centers had a higher percentage of females $(\mathrm{P}=0.019)$, degenerative aortic disease $(\mathrm{P}<0.001)$, and concomitant peripheral artery disease $(\mathrm{P}<0.001)$. With respect to the time period of the study, a significantly increased number of chronic aortic dissection patients $(\mathrm{P}=0.027)$ and redo surgeries $(\mathrm{P}=0.003)$ were recorded during the $1^{\text {st }}$ time period.

\section{Intraoperative results}

Right axillary artery cannulation $(69.6 \%)$ and bilateral selective cerebral perfusion (94.0\%) were performed in the majority of patients and more often in high-volume centers $(\mathrm{P}=0.048$ and $\mathrm{P}=0.004$, respectively, Table 2). Femoral artery cannulation $(3.4 \%)$ was more frequent in lowvolume centers $(\mathrm{P}=0.031)$. Patients in high-volume centers more commonly underwent en-bloc reimplantation of the brachiocephalic trunk and left carotid artery $(\mathrm{P}<0.001)$, as well as a valve-sparing aortic root repair $(\mathrm{P}<0.001)$ and concomitant coronary artery revascularization $(\mathrm{P}=0.042)$. Duration of cardiopulmonary bypass $(\mathrm{CPB})(\mathrm{P}=0.004)$, cardioplegic arrest $(\mathrm{P}=0.002)$, selective antegrade cerebral perfusion $(\mathrm{SACP})(\mathrm{P}<0.001)$ and intraoperative visceral ischemia $(\mathrm{P}<0.001)$ were significantly lower in high- versus low-volume centers. 


\begin{tabular}{|c|c|c|c|c|c|c|c|}
\hline Patients & Overall $(\mathrm{N}=1,165)$ & Low $(\mathrm{N}=334)$ & High $(\mathrm{N}=831)$ & $\mathrm{P}$ & First $(\mathrm{N}=385)$ & Second $(N=780)$ & $\mathrm{P}$ \\
\hline \multicolumn{8}{|l|}{ Mortality } \\
\hline 30 days & $142[12]$ & $51[15]$ & $91[11]$ & 0.048 & $51[13]$ & $91[12]$ & 0.447 \\
\hline Stroke & $83[7]$ & $22[7]$ & $61[7]$ & 0.707 & $38[10]$ & $45[6]$ & 0.015 \\
\hline Permanent & $61[5]$ & $13[4]$ & $48[6]$ & 0.244 & $25[7]$ & $36[5]$ & 0.208 \\
\hline Temporary & $34[3]$ & $13[4]$ & $21[2]$ & 0.247 & $14[4]$ & 20 [3] & 0.355 \\
\hline Spinal cord ischemia & $77[7]$ & $24[7]$ & $53[6]$ & 0.604 & $32[8]$ & $45[6]$ & 0.104 \\
\hline Cardiac low output & $122[11]$ & $47[14]$ & $75[9]$ & 0.015 & 47 [12] & $75[10]$ & 0.186 \\
\hline Respiratory insufficiency & 329 [28] & 82 [25] & $247[30]$ & 0.084 & $102[27]$ & 227 [29] & 0.369 \\
\hline Renal replacement therapy & 252 [22] & $61[18]$ & $191[23]$ & 0.083 & $101[26]$ & $151[19]$ & 0.008 \\
\hline Visceral organs & 33 [3] & $10[3]$ & $23[3]$ & 0.846 & $13[3]$ & $20[3]$ & 0.455 \\
\hline Peripheral ischemia & 35 [3] & $12[4]$ & $23[3]$ & 0.452 & $15[4]$ & $20[3]$ & 0.208 \\
\hline Re-exploration for bleeding & $151[13]$ & $54[16]$ & $97[12]$ & 0.043 & $55[14]$ & 96 [12] & 0.355 \\
\hline
\end{tabular}

Data are presented as $\mathrm{n}[\%]$.

In the $2^{\text {nd }}$ time period, the number of right axillary artery cannulation increased in comparison to the $1^{\text {st }}$ time period $(\mathrm{P}=0.008)$. In addition, the number of direct cannulation of the proximal aorta decreased significantly $(\mathrm{P}<0.001)$ and the number of other cannulation techniques (e.g., brachiocephalic trunk, carotid artery) increased $(\mathrm{P}=0.016)$. In $2^{\text {nd }}$ time period, bilateral selective cerebral perfusion remained the most frequent technique for cerebral protection. Proximally, more ascending aorta interventions $(\mathrm{P}<0.001)$ and aortic valve sparing procedures $(\mathrm{P}<0.001)$ were performed in the $2^{\text {nd }}$ time period. With regard to intraoperative times, visceral ischemia time decreased significantly in the $2^{\text {nd }}$ time period $(\mathrm{P}<0.001)$.

\section{Postoperative results}

Thirty-day mortality was significantly lower in high-volume centers $(11.0 \%$ vs. $15.3 \%, \mathrm{P}=0.048)$, as was in-hospital mortality (13.7\% vs. $18.6 \%, \mathrm{P}=0.046$, Table 3). The total incidence of cerebral (7.1\%) and spinal cord complications (6.6\%) was statistically similar in both groups. Of these, permanent cerebral and spinal cord complications occurred in $5.2 \%$ and $3.9 \%$, respectively. In high- versus low-volume centers, the incidence of postoperative cardiac failure $(9.0 \%$ vs. $14.1 \%, \mathrm{P}=0.015)$ and re-exploration for bleeding $(11.7 \%$ vs. $16.2 \%, \mathrm{P}=0.043)$ was significantly lower in high-volume centers. Other postoperative complications including renal, pulmonary, visceral and peripheral complications were statistically similar in both groups.

In the $2^{\text {nd }}$ versus $1^{\text {st }}$ time period, 30-day mortality $(12 \%$ vs. $13 \%, \mathrm{P}=0.447)$ and in-hospital mortality ( $14 \%$ vs. $17 \%$, $\mathrm{P}=0.258)$ were statistically similar. Cerebral complications decreased significantly in the $2^{\text {nd }}$ time period $(5.8 \%$ vs. $9.9 \%, \mathrm{P}=0.015)$. In addition, the incidence of permanent (3.2\%) and temporary (3.1\%) spinal cord ischemia-related symptoms was numerically lower in the $2^{\text {nd }}$ time period, but not significantly. The incidence of postoperative renal replacement therapy (19.4\%) decreased significantly in the $2^{\text {nd }}$ time period $(\mathrm{P}=0.008)$.

The estimated 10 -year overall survival for the entire group of patients was $46.6 \%$ (Figure $1 A, B, C, D$ ). At 6 years, survival was poorer in low-volume $(56.1 \%) v s$. highvolume (63.3\%) centers (HR, 1.332; 95\% CI, 1.060-1.675; $\mathrm{P}=0.014)$, Figure $2 A$, and was statistically similar between 

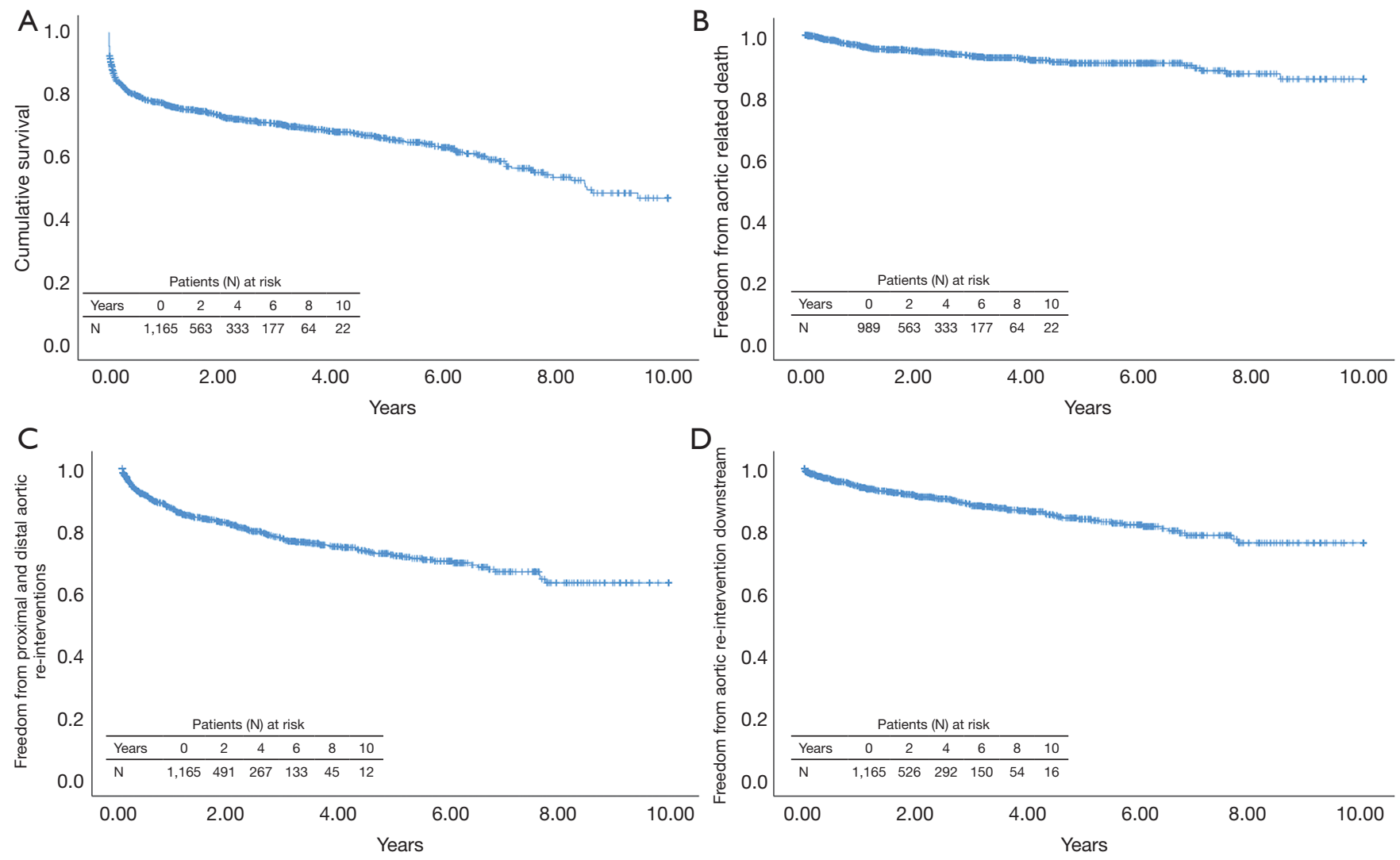

Figure 1 Kaplan-Meier analysis for overall cumulative survival (A), freedom from aortic related death (B) and freedom from proximal and distal aortic re-interventions $(\mathrm{C})$; the freedom from aortic re-interventions along the descending aorta distal to the graft is presented separately in (D).

the $1^{\text {st }}(60.6 \%)$ and $2^{\text {nd }}(66.5 \%)$ time periods (HR, 1.154; 95\% CI, 0.924-1.1442; $\mathrm{P}=0.206)$, Figure $2 B$. During followup, 46 patients $(4.6 \%)$ succumbed to a verified proximal or distal aortic-related complication, and in $19(1.9 \%)$ patients the cause of death was unknown. The overall freedom from aortic-related death at 10 years was therefore $85.7 \%$ (Figure 1B). The freedom from aortic-related death at 6 years was similar in high-volume $(90.5 \%)$ vs. low-volume (92.5\%) centers (HR, 1.058; 95\% CI, 0.579-1.936; $\mathrm{P}=0.854)$, as well as in the $1^{\text {st }}(91.0 \%)$ and $2^{\text {nd }}(91.3 \%)$ periods (HR, 0.935; 95\% CI, 0.546-1.600; $\mathrm{P}=0.806$ ).

Postoperatively, $207(17.8 \%)$ of 1,165 patients underwent at least one secondary intervention; 24 (2.1\%) in the proximal aorta and $182(15.6 \%)$ in the downstream aorta. Indication for proximal aortic surgery was aortic valve regurgitation in $13(1.1 \%)$, infectious endocarditis in $7(0.6 \%)$ and aortic root false aneurysm in $4(0.3 \%)$. Re-interventions in the distal aorta were performed by open surgery in $59(5 \%)$ and/or endovascular surgery in
$130(11.2 \%)$. In 70 of $182(6.0 \%)$ patients, the distal reintervention followed as a second procedure of a planned two-staged treatment. Of the other 112 patients, 90 (7.7\%) underwent a second intervention for a progressive residual aortic pathology and $22(1.9 \%)$ for a new aortic finding including distal stent graft-induced new entries and endoleaks, aortic rupture, and aortic fistula. At 10 years, the overall freedom from aortic re-intervention was $63.2 \%$ (Figure 1C) and the freedom from aortic re-interventions downstream for progressive or new aortic findings was $76.0 \%$ (Figure 1D). Freedom from re-interventions was statistically similar between high- versus low-volume centers and between the $1^{\text {st }}$ and $2^{\text {nd }}$ time periods of the study (Figure 2C,D,E,F).

\section{Discussion}

The FET technique enables the extension of the aortic arch treatment into the descending aorta by implementation of 

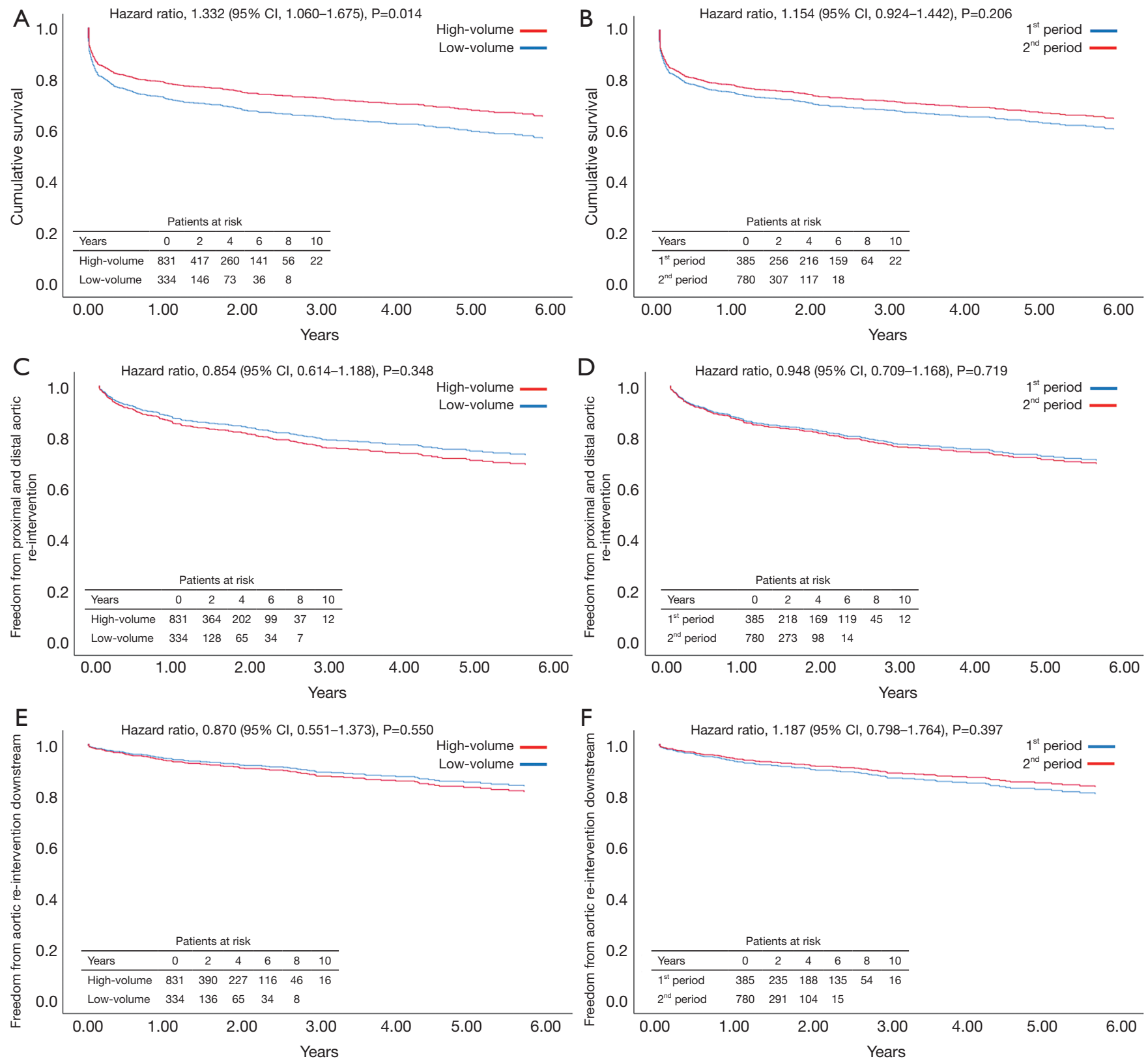

Figure 2 Cumulative survival and freedom from re-interventions at 6 years follow-up between the high- versus low-volume centers (A,C) and between the $1^{\text {st }}$ and $2^{\text {nd }}$ period of the study $(B, D)$; the freedom from re-interventions along the descending aorta distal to the graft is presented separately in $(\mathrm{E}, \mathrm{F})$, respectively.

an endovascular stent graft to a classic surgical prosthesis. FET can be applied to almost all multisegmental thoracic aortic pathologies, with the exception of descending aortic free rupture, mycotic aneurysms, and severe aortic isthmus stenosis (2). Besides the option to treat a distally extended aortic arch disease in one stage via a single median sternotomy, FET allows the durable exclusion of the proximal segment of an otherwise extensive thoracic/ thoracoabdominal aortic disease. FET also facilitates a second endovascular aortic treatment downstream with the covered stent component of the FET prosthesis, representing a safe and reliable proximal landing zone for 
TEVAR (12). Furthermore, the deployment of FET in the mid descending aortic segment facilitates a second open thoracoabdominal approach, since aortic clamping can be positioned at the easily accessible proximal descending aorta and far away from the more complicated transition zone of the distal aortic arch (13).

In acute aortic dissection, the FET stent graft supports the true lumen and the surgical anastomosis from inside, improving hemostasis and reducing late anastomosisrelated complications like pseudoaneurysms and new distal anastomotic entry sites. The false lumen exclusion in aortic dissection by the stent graft results in positive remodeling and resorption of the false lumen thrombus proximally, as well as stabilization of the true lumen distally, in the majority of patients $(14,15)$. This effect is similar to endovascular stent graft therapy after exclusion of the primary proximal entry tear in the descending aorta $(16,17)$. However, in case of FET and in contrary to the classic endovascular treatment, this positive aortic remodeling is achieved without the otherwise required oversizing of the stent graft (7). In addition, a circumferential running suture fixes the FET-stent graft permanently, so that the risk of stent graft migration and proximal endoleak is minimized. Furthermore, this surgical fixation enables the deployment of the stent graft in each part of the aortic arch without the prerequisite of a suitable proximal landing zone, as in classic endovascular treatment. Thus, FET can be used not only in acute type $\mathrm{A}$ aortic dissection, but also in non-A-non-B as well as type $\mathrm{B}$ aortic dissections with a limited proximal landing zone for TEVAR therapy $(18,19)$.

Although the advantages of FET for the treatment of multisegmental thoracic aortic disease are well known, the use of FET as standard treatment for such patients remains controversial $(20,21)$. FET is a major invasive operation and is associated with substantial morbidity and mortality. The treatment requires the use of extracorporeal circulation and hypothermic circulatory arrest, and the duration of surgery is prolonged. Thus, proper patient selection is mandatory. In particular, frail patients or patients with multiple comorbidities, such as severe pulmonary lung disease and generalized peripheral atherosclerotic disease, are associated with an increased mortality risk $(22,23)$. In addition, the experience of surgeons and institutions in dealing with complex aortic arch and descending aorta pathologies, as well as aortic emergencies, may influence postoperative outcomes. Thus, the first aim of the study was the evaluation of patients' outcomes after FET surgery in association with the institutional FET experience.
The second aim of the study was the evaluation of results according to longitudinal increasing experience with FET over time.

The first commercially available FET-prosthesis was the E-vita Open, which was introduced in 2005. The observation of an increased risk of major complications, such as spinal cord ischemia, led to the evolution of surgical techniques in order to reduce surgical trauma, to improve distal organ protection, and to increase the safety of FET deployment (24). In addition, observations about the fate of the residual descending aorta disease after FET have enabled a safer estimation of proper graft sizing for these operations. In order to achieve both of the above-mentioned objectives, data from the International E-vita Open registry were analyzed according to the frequency and number of FET procedures performed in participating centers, as well as comparisons between the first and second periods of the study.

Our results demonstrate significant differences between low- and high-volume centers with regard to patient selection, concomitant procedures, intraoperative techniques, and postoperative outcomes. Low-volume centers operated on a higher proportion of patients with degenerative aortic aneurysmal disease, and high-volume centers operated on more patients with acute aortic dissection and aortic regurgitation. A difference in patients' age was therefore noted, with an older average patient age in low-volume centers compared to high-volume centers. Although the use of FET in acute aortic dissection is controversial, our results demonstrate that the frequency of FET application in aortic emergencies increases with institutional experience. In addition, heterogeneous comorbidities were noticed between centers. The increased frequency of patients with chronic pulmonary and renal disease in high-volume centers also demonstrates the tendency to apply FET treatment in patients with a high risk for postoperative complications and prolonged intensive care treatment. Otherwise, peripheral artery disease was more common in low-volume centers and probably related to the increased number of patients with degenerative aortic disease. Right axillary artery cannulation and bilateral selective cerebral perfusion is felt to be the technique of choice for perfusion and cerebral protection in open aortic arch surgery. However, both techniques were used more frequently in high-volume centers. Although the incidence of adjunct aortic valve-sparing root repair and coronary artery revascularization was significantly increased in highvolume centers, the operative times were significantly lower. 
This observation confirms the association between duration of surgery and institutional experience with complex aortic disease. The positive impact of centers' experience with FET treatment was also demonstrated by the significantly decreased 30 day and in-hospital mortality. Treatment in low-volume centers was associated with an increased number of cardiac complications and re-explorations for bleeding. In contrary, the incidence of postoperative temporary renal replacement was more common in highvolume centers. Finally, neurological cerebral and spinal cord-related complications were statistically similar in both groups.

The chronological analysis demonstrated a shift of FET application towards more acute and significantly less chronic aortic dissections. The less favorable aortic remodeling in chronic dissection and emergence of new endovascular techniques for the treatment of a chronic dissected aortic arch could be reasons for this observation $(3,25)$. However, institutional policies of the centers with regard to patient selection and choice of FET graft may influence this result. The reduced number of redo-surgeries and chronic aortic dissections in $2^{\text {nd }}$ time period explains the increased frequency of ascending aorta replacement in comparison to the $1^{\text {st }}$. In the $2^{\text {nd }}$ time period, the increased use of unilateral cerebral perfusion and other artery cannulation techniques may express a tendency for a more patient-tailored approach. With regard to operative times, the duration of distal hypothermic circulatory arrest distally decreased significantly in the $2^{\text {nd }}$ time period. The main cause for this reduction was the implementation of distal body selective perfusion during the arch repair in some centers. In addition, a significant reduction of renal replacement therapy occurred.

The observed mortality rates were similar between time periods. However, in the $2^{\text {nd }}$ time period, a significant reduction of cerebral complications as well as reduction of spinal cord complications was observed. The reduction of the E-vita Open stent graft length from 15 to $13 \mathrm{~cm}$ and the implementation of distal body selective perfusion during the study period may have contributed to the observed reduction of spinal cord complications $(23,25)$. Nowadays, FET surgery can be applied with similar results in comparison to classic open aortic arch surgery and with a less residual risk for spinal cord complication, close to that observed during endovascular stent graft therapy $(26,27)$.

Our study demonstrates improved results of FET treatment over time. In addition, increasing experience in high-volume centers was associated with an improved survival in follow-up. Considering the high-risk patient population with aortic emergencies including those with extensive aneurysmal disease, our observed mortality rate and survival is acceptable. Although endovascular technology is working towards zone 0 treatment (28), the vast majority of aortic pathologies presented in the current study are not yet suitable for endovascular treatment. Restrictions such as absent or short proximal landing zones in the aneurysmatic and dissected ascending aorta have led to slow progress in this regard.

The freedom of aortic-related death in the current study was $85 \%$, indicating an ongoing risk of severe complications such as aortic rupture and aortic fistula. Considering the proximal complications associated with the aortic valve and aortic root, compromises in proximal surgical repair due to the extension of the aortic arch surgery should be avoided. Downstream, new aortic pathologies and progress of the residual disease may occur, so close follow-up with repeated aortic imaging is strongly recommended.

The results of the International E-vita Open Registry demonstrate that FET surgery has improved over the years, and with increasing institutional experience. Although our study is limited by its retrospective design, it is one of the largest studies to date on this procedure. The lack of a standardized surgical protocol between centers is another potential weakness of the study, but this heterogeneity also allows for analysis of "real-world" practice. Our data also demonstrate that an institutional learning curve exists and needs to be overcome, ideally by a specialized, dedicated aortic team.

\section{Acknowledgments}

None.

\section{Footnote}

Conflicts of Interest: $\mathrm{HJ}$ was the consultant to Jotec $\mathrm{GmbH}$ during the study period. The other authors have no conflicts of interest to declare.

Open Access Statement: This is an Open Access article distributed in accordance with the Creative Commons Attribution-NonCommercial-NoDerivs 4.0 International License (CC BY-NC-ND 4.0), which permits the noncommercial replication and distribution of the article with the strict proviso that no changes or edits are made and the original work is properly cited (including links to both the 
formal publication through the relevant DOI and the license). See: https://creativecommons.org/licenses/by-nc-nd/4.0/.

\section{References}

1. Karck M, Kamiya H. Progress of the treatment for extended aortic aneurysms; is the frozen elephant trunk technique the next standard in the treatment of complex aortic disease including the arch? Eur J Cardiothorac Surg 2008;33:1007-13.

2. Tsagakis K, Wendt D, Dimitriou AM, et al. The frozen elephant trunk treatment is the operation of choice for all kinds of arch disease. J Cardiovasc Surg (Torino) 2018;59:540-6.

3. Shrestha M, Martens A, Kaufeld T, et al. Single-centre experience with the frozen elephant trunk technique in 251 patients over 15 years. Eur J Cardiothorac Surg 2017;52:858-66.

4. Preventza O, Liao JL, Olive JK, et al. Neurologic complications after the frozen elephant trunk procedure: a meta-analysis of more than 3000 patients. J Thorac Cardiovasc Surg 2019. [Epub ahead of print].

5. Inoue $\mathrm{Y}$, Matsuda $\mathrm{H}, \mathrm{Omura} \mathrm{A}$, et al. Comparative study of the frozen elephant trunk and classical elephant trunk techniques to supplement total arch replacement for acute type A aortic dissection†. Eur J Cardiothorac Surg 2019;56:579-86.

6. Jakob H, Tsagakis K, Leyh R, et al. Development of an integrated stent graft-dacron prosthesis for intended one-stage repair in complex thoracic aortic disease. Herz 2005;30:766-8.

7. Tsagakis K, Jakob H. Which frozen elephant trunk offers the optimal solution? Reflections from Essen group. Semin Thorac Cardiovasc Surg 2019;31:679-85.

8. Tsagakis K, Dohle D, Benedik J, et al. Overall Essen's experience with the E-vita open hybrid stent graft system and evolution of the surgical technique. Ann Cardiothorac Surg 2013;2:612-20.

9. Leone A, Di Marco L, Coppola G, et al. Open distal anastomosis in the frozen elephant trunk technique: initial experiences and preliminary results of arch zone 2 versus arch zone 3†. Eur J Cardiothorac Surg 2019;56:564-71.

10. Weiss G, Santer D, Dumfarth J, et al. Evaluation of the downstream aorta after frozen elephant trunk repair for aortic dissections in terms of diameter and false lumen status. Eur J Cardiothorac Surg 2016;49:118-24.

11. Svensson LG. Rationale and technique for replacement of the ascending aorta, arch, and distal aorta using a modified elephant trunk procedure. J Card Surg 1992;7:301-12.

12. Haensig M, Schmidt A, Staab H, et al. Endovascular repair of the thoracic or thoracoabdominal aorta following the frozen elephant trunk procedure. Ann Thorac Surg 2020;109:695-701.

13. Folkmann S, Weiss G, Pisarik H, et al. Thoracoabdominal aortic aneurysm repair after frozen elephant trunk procedure. Eur J Cardiothorac Surg 2015;47:115-9; discussion 119.

14. Dohle DS, Tsagakis K, Janosi RA, et al. Aortic remodelling in aortic dissection after frozen elephant trunk $†$. Eur J Cardiothorac Surg 2016;49:111-7 .

15. Iafrancesco M, Goebel N, Mascaro J, et al. Aortic diameter remodelling after the frozen elephant trunk technique in aortic dissection: results from an international multicentre registry. Eur J Cardiothorac Surg 2017;52:310-8.

16. Leshnower BG, Duwayri YM, Chen EP, et al. Aortic remodeling after endovascular repair of complicated acute type B aortic dissection. Ann Thorac Surg 2017;103:1878-85.

17. Sultan I, Siki MA, Bavaria JE, et al. Predicting distal aortic remodeling after endovascular repair for chronic DeBakey III aortic dissection. Ann Thorac Surg 2018;105:1691-6.

18. Shrestha M, Bachet J, Bavaria J, et al. Current status and recommendations for use of the frozen elephant trunk technique: a position paper by the Vascular Domain of EACTS. Eur J Cardiothorac Surg 2015;47:759-69.

19. Czerny M, Schmidli J, Adler S, et al. Current options and recommendations for the treatment of thoracic aortic pathologies involving the aortic arch: an expert consensus document of the European Association for CardioThoracic surgery (EACTS) and the European Society for Vascular Surgery (ESVS). Eur J Cardiothorac Surg 2019;5 5:133-62.

20. Lansman SL, Goldberg JB, Kai M, et al. Extended arch procedures for acute type A aortic dissection: a downstream problem? Semin Thorac Cardiovasc Surg 2019;31:17-20.

21. Di Bartolomeo R, Leone A, Di Marco L, et al. When and how to replace the aortic arch for type A dissection. Ann Cardiothorac Surg 2016;5:383-8 .

22. Jakob H, Dohle D, Benedik J, et al. Long-term experience with the E-vita Open hybrid graft in complex thoracic aortic disease†. Eur J Cardiothorac Surg 2017;51:329-38.

23. Leontyev S, Tsagakis K, Pacini D, et al. Impact of clinical factors and surgical techniques on early outcome of patients treated with frozen elephant trunk technique by using EVITA open stent-graft: results of a multicentre 
study. Eur J Cardiothorac Surg 2016;49:660-6.

24. Tsagakis K, Dohle DS, Wendt D, et al. Left subclavian artery rerouting and selective perfusion management in frozen elephant trunk surgery. Minim Invasive Ther Allied Technol 2015;24:311-6.

25. Pacini D, Tsagakis K, Jakob H, et al. The frozen elephant trunk for the treatment of chronic dissection of the thoracic aorta: a multicenter experience. Ann Thorac Surg 2011;92:1663-70; discussion 1670.

26. Urbanski PP, Luehr M, Di Bartolomeo R, et al. Multicentre analysis of current strategies and outcomes

Cite this article as: Tsagakis K, Pacini D, Grabenwöger M, Borger MA, Goebel N, Hemmer W, Laranjeira Santos A, Sioris T, Widenka K, Risteski P, Mascaro J, Rudez I, Zierer A, Mestres CA, Ruhparwar A, Di Bartolomeo R, Jakob H. Results of frozen elephant trunk from the international E-vita Open registry. Ann Cardiothorac Surg 2020;9(3):178-188. doi: 10.21037/acs-2020fet-25 in open aortic arch surgery: heterogeneity is still an issue. Eur J Cardiothorac Surg 2016;50:249-55.

27. Dijkstra ML, Vainas T, Zeebregts CJ, et al. Editor's choice - spinal cord ischaemia in endovascular thoracic and thoraco-abdominal aortic repair: review of preventive strategies. Eur J Vasc Endovasc Surg 2018;55:829-41.

28. Spear R, Haulon S, Ohki T, et al. Editor's choice - subsequent results for arch aneurysm repair with inner branched endografts. Eur J Vasc Endovasc Surg 2016;51:380-5. 